\title{
A New Method for Determining the Magnetic Properties of Solid Materials Employed in Unconventional Magnetic Circuits
}

\author{
Ahmed M. Mohammed, Tom Cox, Member, IEEE, Michael Galea, Member, IEEE, \\ and Chris Gerada, Fellow Member, IEEE
}

\begin{abstract}
To evaluate the quality of magnetic materials used in electrical machines, accurate material characterization is required. For common, solid (nonlaminated) ferromagnetic materials, characterization procedures such as the toroidal ring sample test method are capable of mapping electro-magnetic properties with reasonable accuracy. This is true when the investigation is for solid materials to be used in conventional magnetic circuits, i.e. where the flux paths and induced eddy currents follow the more common 'radial' characteristics, as in a standard rotating machine.

When solid ferromagnetic materials are employed in unconventional machine structures, such as for transverse flux machines or tubular linear machines, classical methods are not capable of achieving an accurate representation of the flux conditions in the machine, thus resulting in inaccurate characterization data that usually underestimates the total loss prediction.

In this paper a new testing method is proposed to impose the correct flux conditions for solid materials (used in tubular linear machines) and accurately map the eddy current losses in the solid parts. The proposed method uses a simple experimental test setup to characterize the power loss of solid, ferro-magnetic material. The basic experimental results from the new setup are compared to results from 3D finite element analysis.
\end{abstract}

Index Terms- Materials characterization, solid materials, toroidal ring test, tubular machine (TLPM), eddy current losses.

Manuscript received December 30, 2015; revised April 20, 2016 and April 04, 2016; accepted May 12, 2016. This work was supported in part by the Clean Sky JTI-Systems for Green Operations ITD.

A. Mohammed, T. Cox, M. Galea and C. Gerada are with the Power Electronics, Machines and Control Group, Department of Electrical and Electronic Engineering, the University of Nottingham, Nottingham, UK (e-mail: eexam44@nottingham.ac.uk; T.Cox@nottingham.ac.uk; Michael.Galea@nottingham.ac.uk; Chris.Gerada@nottingham.ac.uk).

\section{INTRODUCTION}

$\mathrm{T}$ RADITIONALLY, the effects of eddy currents and iron losses in electrical machines are reduced by employing electrically insulated laminations [1]. However, certain types of machines, such as tubular linear machines [2] can actually benefit from having solid structures for their stator and mover cores. As shown in [2] and [3], due to the particular 3D flux directions and complex manufacturing aspects in tubular machines, a solid structure of stator core can actually represent an optimal solution in terms of performance vs. cost. While this comes at the cost of extra iron losses [3], there are a number of methods and techniques that can be implemented to mitigate and reduce these core loss effects [3]. These procedures however require accurate mapping of the material characteristics in order to achieve an optimal machine design, which due to the unconventional magnetic circuits cannot be achieved by the standard procedures mentioned in [4] and [5]. In this paper, a new method for the characterization of solid magnetic material, with focus on the core power losses is proposed and investigated. This method involves a simple experimental set-up capable of characterizing solid materials to be used in non-standard magnetic circuits such as that of the tubular machine shown in Fig. 5, where the flux travels axially in the stator yoke. In order to further validate the proposed setup, a high-tech, magnetic measurement system, MPG 200, Brockhaus, is also used and compared with $3 \mathrm{D}, \mathrm{FE}$ results achieved with time harmonic and transient solvers [6].

\section{Material Characterization Methods Overview}

This section introduces material characterization methods for laminated and for solid materials and highlights the limitations regarding such procedures when applied to systems that have unconventional magnetic circuits.

\section{A. Laminated materials}

Today, there is a wealth of literature and data that details the magnetic properties of laminated materials [1]. Alternatively, fast and accurate testing methods for such materials can be achieved by implementing 'simple' tests such as the Epstein 
frame method and the single sheet tester method. The toroidal ring method allows stacks of lamination material in the form of circular ring samples to be characterized quite accurately. In some cases, the complete machine stator is used instead of a toroidal ring sample [4] as shown in Fig. 1.

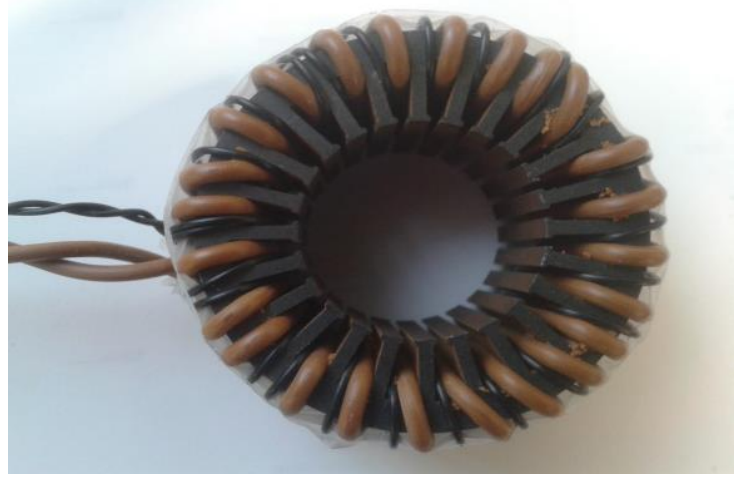

Fig. 1. Material characterization: lamination stacks.

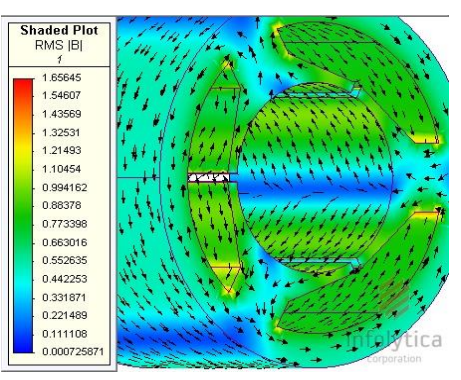

a) Main flux paths

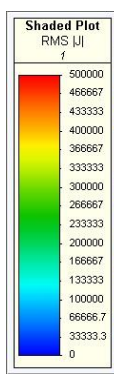

b) Eddy currents
Fig. 2. Standard magnetic circuits of rotating machines

\section{B. Solid materials for conventional arrangements}

In terms of solid magnetic materials, today there are considerable amounts of freely-available data regarding the mechanical and physical properties of such materials as EN8, SSJ416, etc. [7]. However, when it comes to the magnetic properties of such solid materials, there is often a lack of information. Even when such data is available, it is generally incomplete and does not provide all the information that the electrical machine designer requires. In order to mitigate this there are a number of testing procedures that can be used for characterizing the ferro-magnetic properties of solid materials.

However such procedures are usually limited to applications where the magnetic circuit is relatively standard such as for conventional rotary 'radial' electrical machines, where the main flux and the eddy currents follow the 'standard' paths such as shown in Fig. 2 (a) and (b) respectively.

\section{Solid materials for unconventional arrangement}

For electrical machines that employ a non-standard configuration such as the tubular machine of Fig. 3, which was explored extensively in [2] and [8], the magnetic circuit is quite different from a standard rotary machine.

In such an electrical machine, the magnetic flux travels in the same direction of the moving parts [9]. Hence, the main flux path becomes 'axial', while the eddy current paths become 'radial' as illustrated in Fig. 4 (a) and (b) respectively.

This indicates that common material characterization methods do not accurately represent the true flux and current conditions in this configuration. If such methods are used, this can result in non-optimal design of solid magnetic material electrical machines.

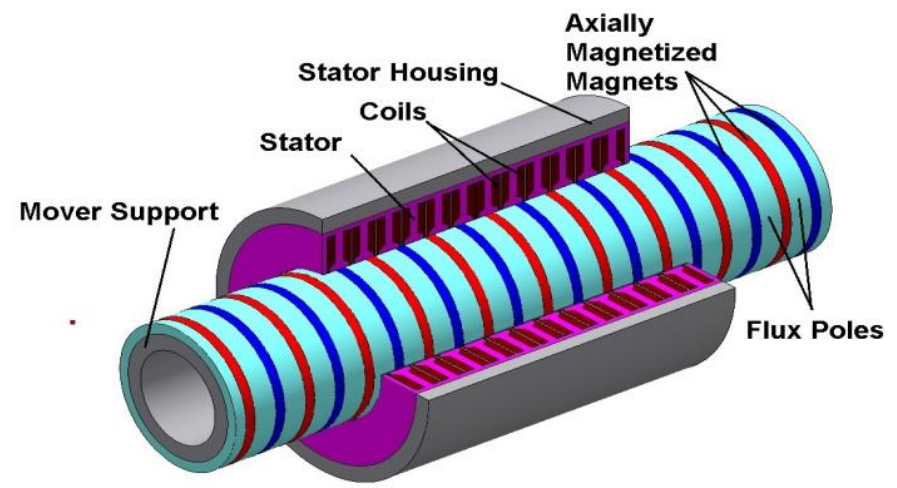

Fig. 3. Non-conventional machine: tubular motor

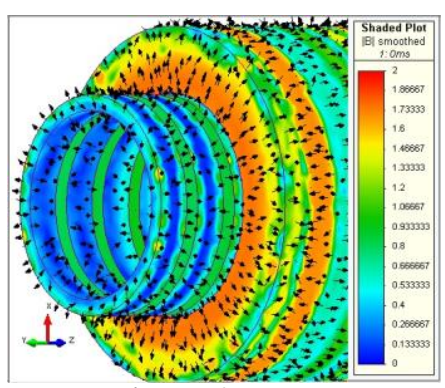

a) Main flux paths

Fig. 4. Non-standard magnetic circuit

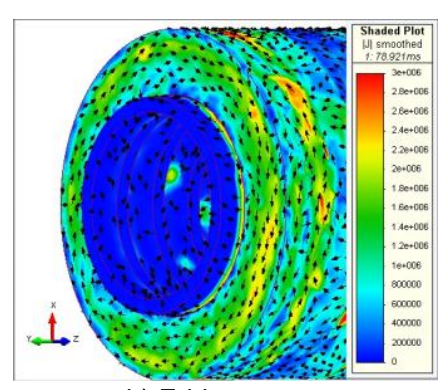

b) Eddy currents

\section{Characterization Method for Conventional MAGNETIC CIRCUITS}

This section illustrates how characterization of material employed in standard magnetic circuits can be achieved. Considering a homogenous material structure, then isotropic magnetic properties can be assumed.

A ring sample setup with two windings as shown in Fig. 5 is required, where the main winding creates the magnetic field intensity $\mathrm{H}$ and the second winding is used to measure the generated flux density B in the ferromagnetic core.

The objective of this test is to calculate the relative permeability $\mu_{r}$ and the specific losses of the material at different frequencies and flux densities. The maximum values of $H$ and $B$ can be computed from (1) and (2), where $V_{\text {sense }}$ is the average or DC value of the output signal measured by an average-type voltmeter. An RMS-type voltmeter could also be used when the form factor of the output voltage of the secondary winding is kept at $\approx 1.1107$ which as discussed in [5], indicates a pure sinusoidal $B$ in the ring sample. Then, $\mu_{r}$ can be calculated with (3) where $\mu_{o}$ is the permeability of free space.

$$
\begin{array}{r}
\hat{H}=\frac{N_{\text {main }} \hat{I}}{l_{\text {core }}} \\
\hat{B}=\frac{v_{\text {sense }}}{4 f N_{\text {sense }}} \\
\mu_{r}=\frac{\hat{B}}{\mu_{o} \hat{H}}
\end{array}
$$


Fig. 6 illustrates a test set-up that involves a toroidal ring core with uniform cross-sectional area, as advised by the BS EN 60404-6:2003 [5].

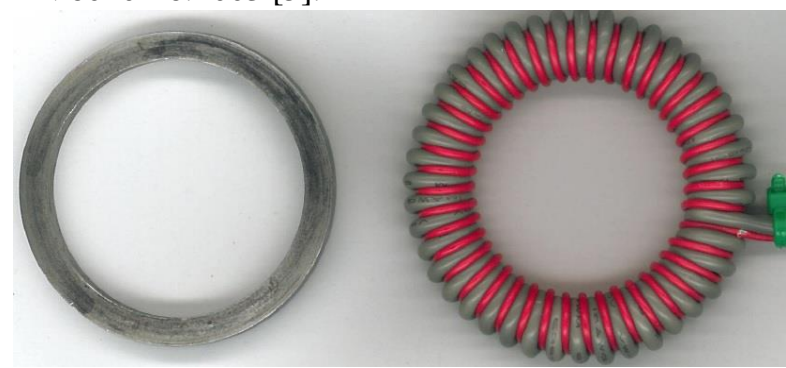

Fig. 6. EN8 solid material ring and toroidal method test set-up

TABLE 1

PARAMETERS OF RING SAMPLE

\begin{tabular}{ll}
\hline \multicolumn{1}{c}{ Quantity } & \multicolumn{1}{c}{ value } \\
\hline Material type & EN8 Solid Steel \\
Inner diameter & $45 \mathrm{~mm}$ \\
Outer diameter & $55 \mathrm{~mm}$ \\
Ring height & $5 \mathrm{~mm}$ \\
Mass & $30 \mathrm{~g}$ \\
Mass density & $7840 \mathrm{~kg} / \mathrm{m}^{3}$ \\
Resistivity $\left(20^{\circ} \mathrm{C}\right)$ & $2.21 \mathrm{e}-007 \Omega / \mathrm{m}$ \\
$N_{\text {main }}$ & 80 \\
$N_{\text {sense }}$ & 75
\end{tabular}

Considering a purely sinusoidal B inside the ring sample, the BH curves of an EN8 solid steel ring sample whose parameters are shown in Table I, is obtained through a closed loop magnetic measurements system (MPG 200D). This is done for a frequency range of 2.5 to $50 \mathrm{~Hz}$, with the resulting curves being shown in Fig.7. The figure also shows the DC magnetic characteristics of EN8 obtained from the MPG 200 measurement system.

Fig. 8 shows the relationship between $\mu \mathrm{r}$ and $\mathrm{B}$ at different applied frequencies for the EN8 sample. It can be clearly observed how $\mu \mathrm{r}$ is strongly frequency dependent and that $\mu \mathrm{r}$ is higher at low frequencies, reaching its maximum values when the flux density is equal to $0.7 \mathrm{~T}$ at $2.5 \mathrm{~Hz}$. Generally, at very low frequencies, the $\mathrm{AC} \mu_{r}$ is approximately identical to the DC value of $\mu_{r}$ [10].

One of the main challenges related to the use of solid materials is the eddy current loss. In this work, the total iron loss Pwatt in the ring sample is measured by using the singlephase wattmeter method. The current coil of the wattmeter is connected in series with the main winding and its voltage coil is connected in parallel with the sense winding. Equation (4) is then used to compute the total core power losses Ploss, where it is important to note that the copper loss has no effect on the wattmeter reading because only the main current is measured in the primary side [5].

$$
P_{\text {loss }}=\frac{N_{\text {main }}}{N_{\text {sense }}} P_{\text {watt }}
$$

As described in [11], the total loss over one electrical cycle can be numerically computed by (5), where $k$ is the sampling number per complete electrical cycle.

$$
P_{\text {loss }}=\frac{N_{\text {main }}}{N_{\text {sense }}} \cdot \frac{1}{K} \sum_{k=1}^{K} i_{\text {main }}[k] \cdot v_{\text {sense }}[k]
$$

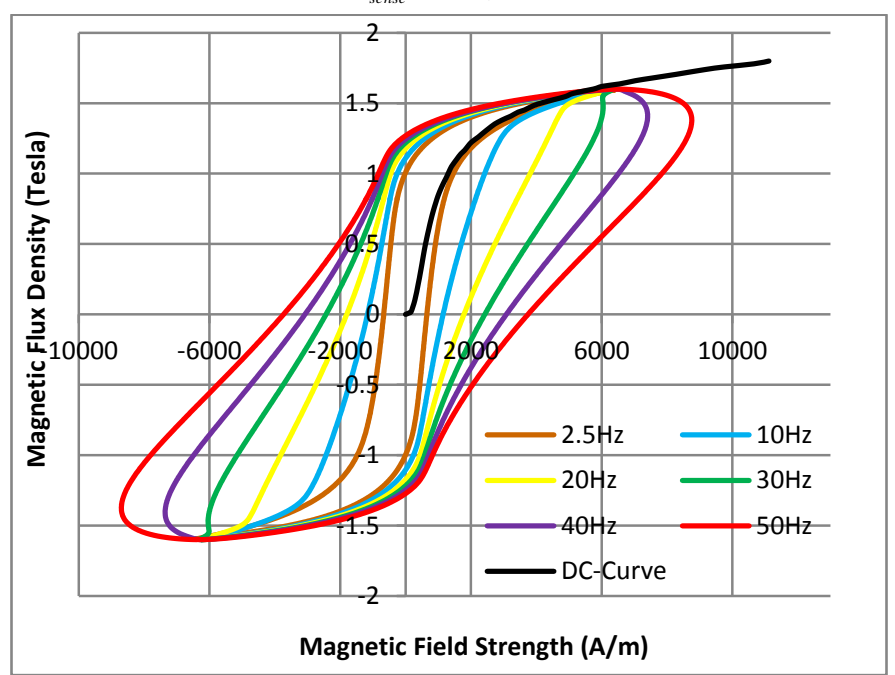

Fig. 7. EN8 material: $\mathrm{BH}$ curves (including $\mathrm{DC}$ ) for different frequencies at $\mathrm{B}=1.6 \mathrm{~T}$

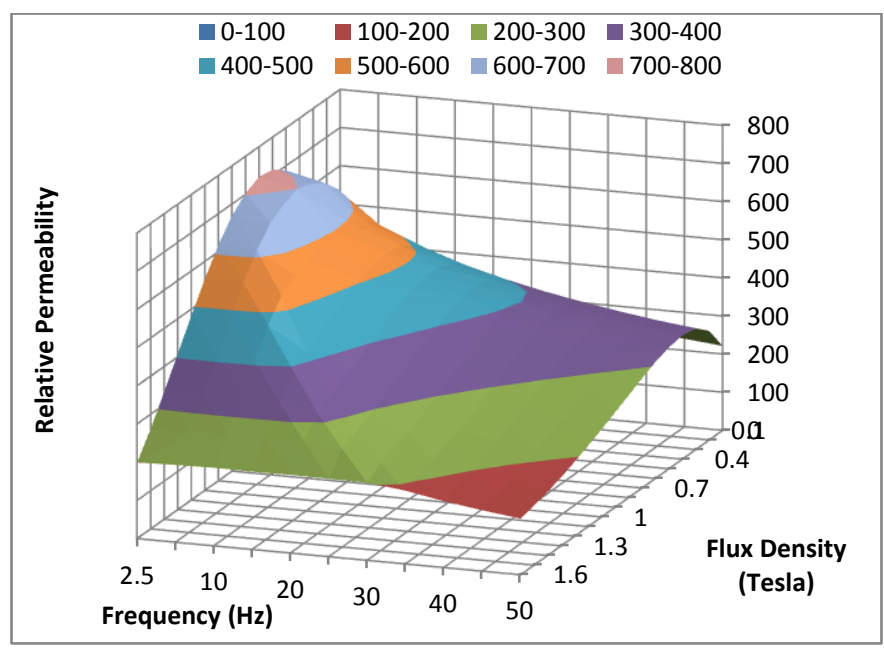

Fig. 8. Relative permeability as a function of frequency and B

The iron losses calculated from (5) can be categorized into three types of losses, namely eddy current losses, hysteresis loss and excess (abnormal) loss. The latter 'abnormal' loss is considered as part of the total eddy current losses [12, 13]. As that the hysteresis losses increase linearly with the applied frequency while the eddy current losses increase with the square of the frequency, these losses can be easily separated by using (6) and (7), where $P_{(f l)}$ is the total iron power loss at frequency $f_{1}, P_{(f 2)}$ is the total iron power losses at frequency $f_{2}$, $P_{e}$ is the eddy current power loss and $P_{h}$ is the hysteresis power loss.

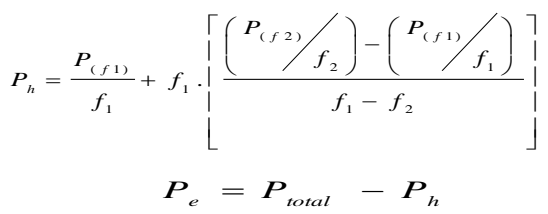


Thus, using all the above, the actual core losses in the ring sample can be identified. For the ring sample discussed above, Fig. 9 shows the total losses derived from experimental tests at different frequencies and flux densities.

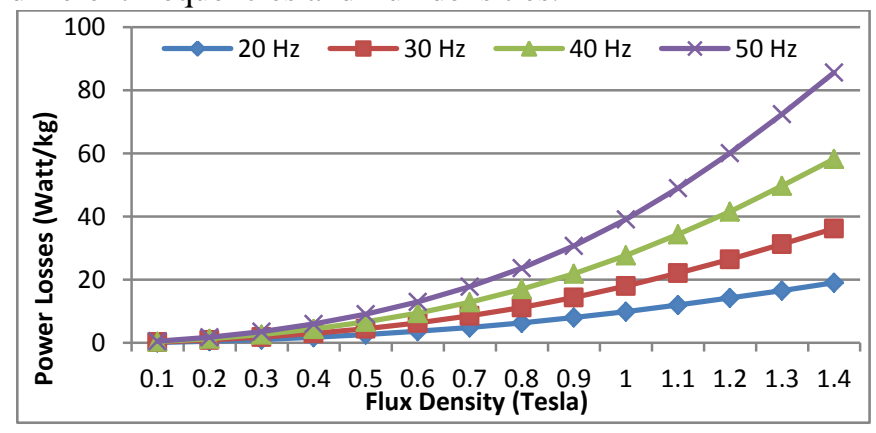

Fig. 9. Power losses of the toroidal ring sample

This method provides accurate measurement of $\mu_{r}$ for magnetic materials forming any closed magnetic circuit. However, measuring $P_{h}$ with this method is only suitable for alternating or one dimensional traveling flux, and $P_{h}$ behaves very differently when two-dimensional or rotating magnetic flux is applied [14]. The generated eddy current, and hence $P_{e}$, behaves completely different if the flux lines pass radially instead of azimuthally. Therefore, one directional inducing flux method, such as shown in Fig. 5, would results in nonoptimal and inaccurate material characterization.

\section{ChaRActerization OF Materials to BE USED IN UNCONVENTIONAL MAGNETIC CIRCUITS}

\section{A. The concept}

The proposed method to characterize materials engaged in unconventional magnetic circuits (such as shown in Fig. 5) is proposed on a relevant ring sample of the material to be used in the final TLPM machine design. The method is mainly based on generating a magnetic flux passing radially through the ring sample as shown in Fig. 10. By combining the belowproposed procedure with the 'standard' toroidal ring test of Fig. 8, it is actually possible to provide complete magnetic characteristics for any magnetic circuit possible from all the known types of electric machine, including rotating machines, axial flux machines and radial flux machines.

To generate the particular field required to emulate the TLPM machine flux pattern, two coils connected in series are accommodated on both sides of the ring sample. The direction of the current flow in the first main coil should have a $180 \mathrm{o}$ phase shift to the second main coil as otherwise only leakage flux would pass through the ring sample.

The above can be mathematically described by using Maxwell's equations. In general, time varying current carrying coils produce an alternating $H$ in the direction which can be identified by the right hand rule. This field induces an eddy current in an electrically conducting magnetic material in the same direction of the current. By expanding Maxwell's equation $(\nabla \times \mathrm{H}=J)$ into cylindrical coordinates, then as shown in [15], this can be mathematically described by (8).

$$
\begin{aligned}
J & =a_{r}\left(\frac{1}{r} \frac{\partial \mathrm{H}_{z}}{\partial \phi}-\frac{\partial \mathrm{H}_{\phi}}{\partial z}\right)+a_{\phi}\left(\frac{\partial \mathrm{H}_{r}}{\partial z}-\frac{\partial \mathrm{H}_{z}}{\partial r}\right) \\
& +a_{z}\left(\frac{1}{r} \frac{\partial\left(r \mathrm{H}_{\phi}\right)}{\partial r}-\frac{1}{r} \frac{\partial \mathrm{H}_{r}}{\partial \phi}\right)
\end{aligned}
$$

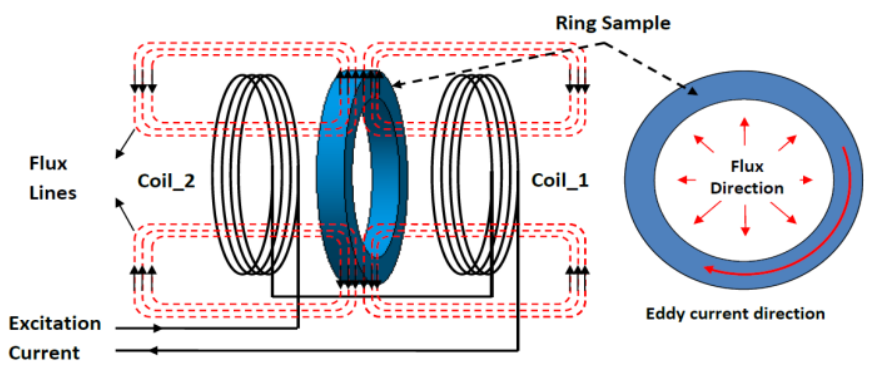

Fig. 10. Ring sample and coils connection diagram with radial flux

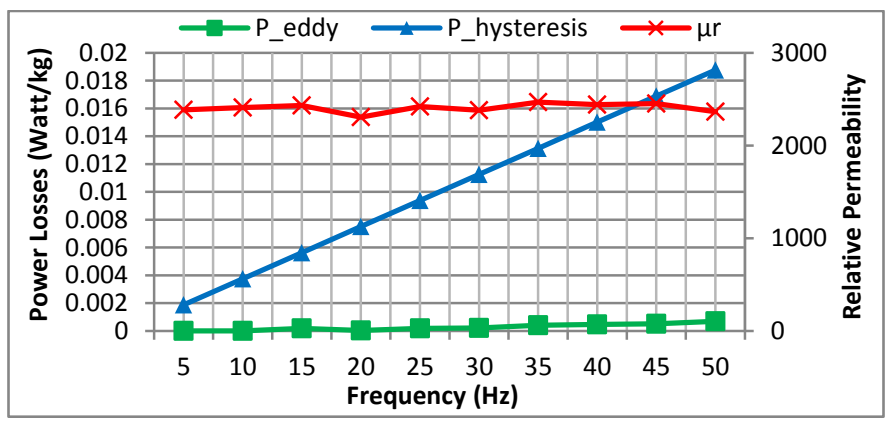

Fig. 11. Magnetic characteristics of Ferrite (0W-48613-TC)

By assuming that the eddy current is rotating in the azimuth direction only, then (8) can be simplified to (9).

$$
J_{\text {ring }}=a_{\phi}\left(\frac{\partial \mathrm{H}_{r, \text { ring }}}{\partial z}-\frac{\partial \mathrm{H}_{z, \text { ring }}}{\partial r}\right)
$$

\section{B. The set-up}

The objective of the proposed set-up is to generate the nonstandard magnetic flux direction described in the previous section. The ring sample is fitted with two primary coils and two secondary coils, as very high current densities are required to obtain the desired $\mathrm{B}$ in the ring. To further aid the increase of magnetic flux for a given current in the main windings, high permeability, ferrite magnetic material $(0 \mathrm{~W}-$ 48613-TC) is used for the frame core. This ferrite material exhibits very low magnetic losses at high frequencies while it enjoys negligible losses at low frequencies [16]. The main challenge with this particular material is its relatively low saturation level, approximately 0.4T. Fig. 11 illustrates the experimentally validated magnetic properties of the ferrite core including its $\mu_{r}$, eddy current losses and the hysteresis losses. The total losses of the ferrite core represent less than $0.02 \%$ of the specimen losses at the highest frequency. Having such low values of losses justifies neglecting the ferrite core losses in the proposed model and experimental setup.

While the general concept shown in Fig. 10 does provide the required flux, it was however felt important to consider certain aspects of the application right from the start. An important aspect of tubular machines is the extra length added to the ends of the stator yoke in order to reduce the cogging force due to the finite edges [17]. The resulting eddy current in 
these parts behaves in a different manner from the rest of the magnetic circuit, mainly due to this part not being completely wound like the machine teeth [18]. Considering this, it was then decided to consider two variations of the proposed set-up, one to consider the teeth and the other to consider the rest of the tubular machine magnetic circuit.

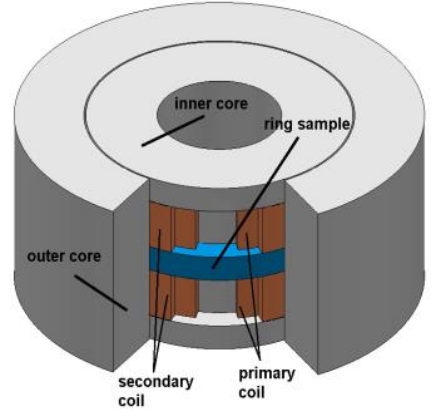

a) The concept

Fig. 12. The proposed Set-up 1

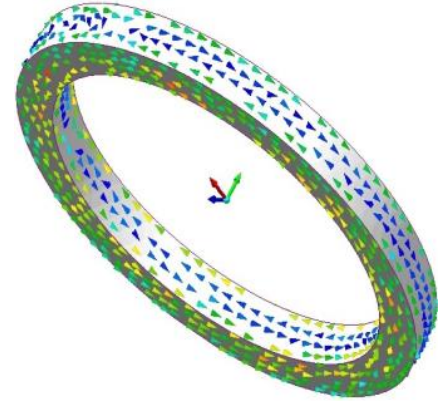

b) Eddy current pattern

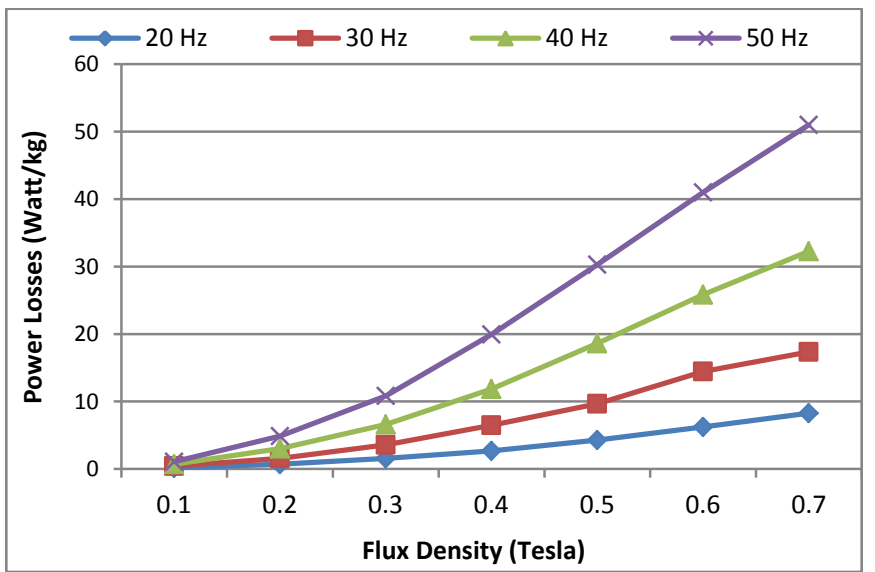

Fig. 13. Set-up 1: Ring power losses from FE model

1) Set-up 1

The first set-up is aimed to characterize the material used for the TLPM machine's teeth. A FE model of the proposed Set-up 1 is shown in Fig. 12a, where the tooth of the TLPM machine is represented by the ring sample. The ring sample is fitted between two primary coils and two secondary coils connected in series. It is important to set the correct polarity of the coils in order to have the mmf of the excitation coils and the back-emf of the sensing coils with a $180^{\circ}$ phase shift between them.

Considering Fig. 5a and Fig. 10, then as is explained in [15] and [18], eddy currents in the ring sample will rotate in two opposite directions following the direction of the excitation current in the adjacent coils on each ring-side. Fig. 12b illustrates the behavior of the generated eddy currents which, as expected, is similar to the TLPM machine eddy current shown in Fig. 5b.

The 3D, FE model is then used to assess the validity of the proposed method for predicting the eddy current loss in the ring sample. The model is solved for a range of frequencies and flux densities, with Fig. 13 showing the resulting eddy current losses as a function of applied frequency and $B$. Considering the above, it is also important to note that with this Set-up 1, extra losses can be incurred due to eddy current pulsations between the two sides of the ring sample, mainly due to the $180^{\circ}$ phase shift between the eddy currents generated in each ringside. This can potentially result in a relatively higher loss than for the toroidal method [18].

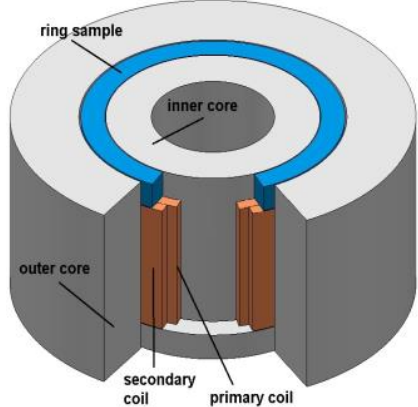

a) The concept
Fig. 14. The proposed Set-up 2

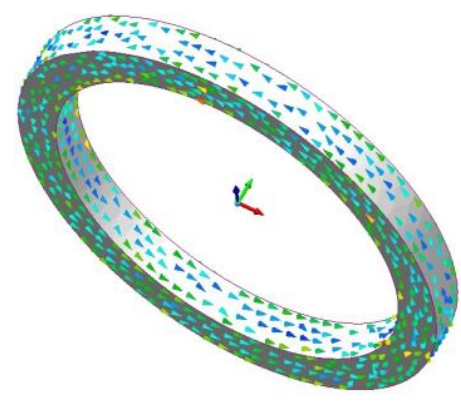

b) Eddy current pattern

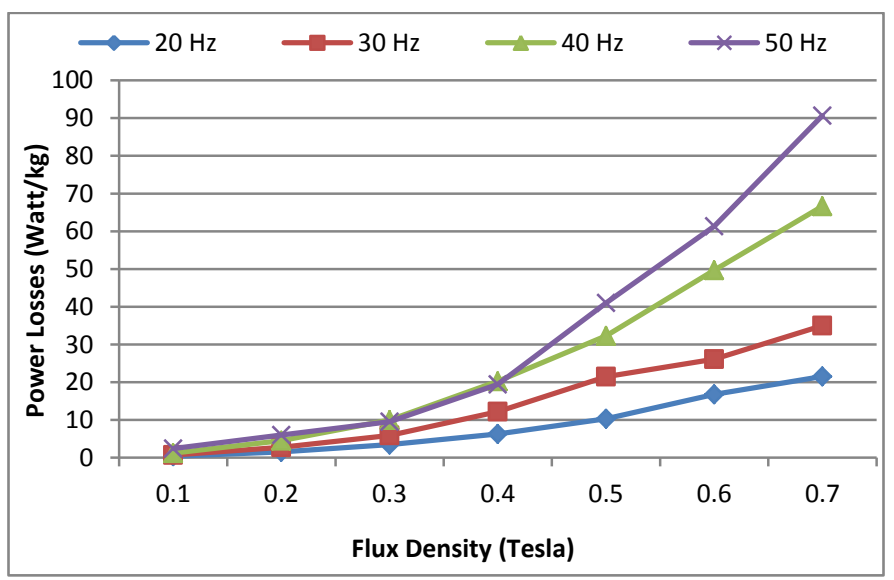

Fig. 15. Set-up 2: Ring power losses from FE model

\section{2) Set-up 2}

The second proposed set-up is used to characterize solid material lying between two coils, carrying currents in the same direction or material with a current carrying coil only on one side. In this case, the eddy current flows in one direction, thus facing lower electrical resistance and rotating free inside the ring sample (see Fig. 14b). In the TLPM motor application, this can represent a single layer winding configuration or the finite edges of the tubular machine. A FE model of the proposed Set-up 2 is shown in Fig. 14a, where it can be observed how the ring sample is no longer positioned between two coils. Fig. 14b shows the behavior of the eddy currents that travel only in one direction.

Figure 15 shows the eddy current losses obtained from this $3 \mathrm{D}$, FE model as a function of applied frequency and $B$. From Fig. 15, it can be observed that the losses in Set-up 2 are significantly higher than those of Set-up 1. This is mainly due to the unidirectional eddy current behavior passing through the larger effective cross sectional area (A) which directly increases the effective conductance of the medium. Considering (10), where $J$ is the eddy current density, $\sigma$ is the conductivity and $\omega$ is the angular speed, then it can be perceived that for a fixed $B$, the eddy current density is 
directly proportional to the electrical conductivity of the material. This means that the eddy loss is also directly proportional to the $A$ of the ring sample, resulting in an increase in the magnitude of the eddy current itself and therefore an increase in power losses.

$$
\nabla \times J=-j \omega \sigma B
$$

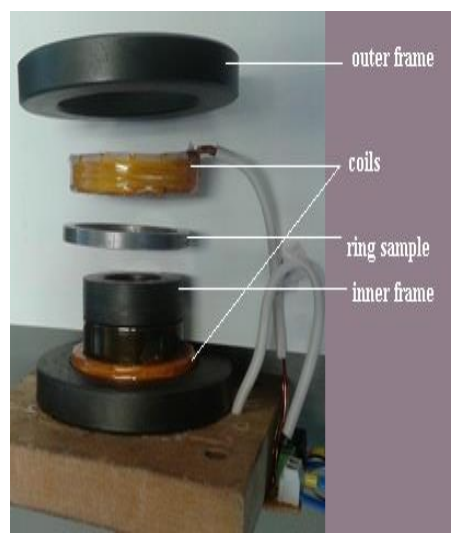

a) Set-up components

Fig. 16. The experimental set-up
(10)

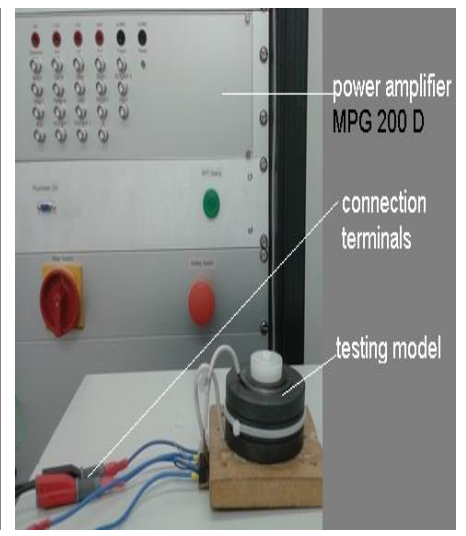

b) Complete set-up

\section{EXPERIMENTAL VALIDATION}

In order to validate the proposed method, a purposely-built experimental set-up is prototyped. This is shown in Fig. 16, where the set-up components can be observed in Fig. 16a and the overall scheme can be observed in Fig. 16b.

The material characterization for the two configurations explained in Section IV is thus performed experimentally on the setups shown in Fig. 16. Both Set-up 1 and Set-up 2 are tested in order to characterize the required material to be used for the design of an optimized TLPM machine.

By applying the methods described in Section III to the experimental results achieved from these tests then the core losses in the samples can be derived.

The core losses experimentally obtained for the 'tooth material' by Set-up 1 are shown in Fig. 17, while Fig. 18 illustrates the core losses of the machine 'edges sections', obtained by Set-up 2.

Fig. 19 compares the results obtained from the 3D, FE models with those achieved experimentally. Excellent similarity is achieved, thus validating the proposed models.

In order to fully appreciate the significance of the proposed methods, a comparison between the resulting eddy current losses from the above mentioned methods is illustrated in Fig. 20. The objective of this is to show that the total eddy current losses resulting from a ring sample that has to operate in a magnetic circuit with an unconventional flux direction are significantly higher than indicated by standard (toroidal) tests.

The classical toroidal method of Fig. 6 results in a very low eddy current loss, while the proposed methods of Fig. 10 indicate a much greater eddy current loss component. This illustrates the significant under-estimation of eddy current losses if traditional methods are used to test and characterize materials being used in unconventional magnetic circuits. Obviously, such an error in the correct estimation of losses can have a severe impact on the design of an electrical machine that makes use of an unconventional magnetic circuit.

Also, Fig. 21 shows the relation between the eddy current losses of all the mentioned methods for different frequencies. This is done for a fixed $\mathrm{B}$ of $0.7 \mathrm{~T}$.

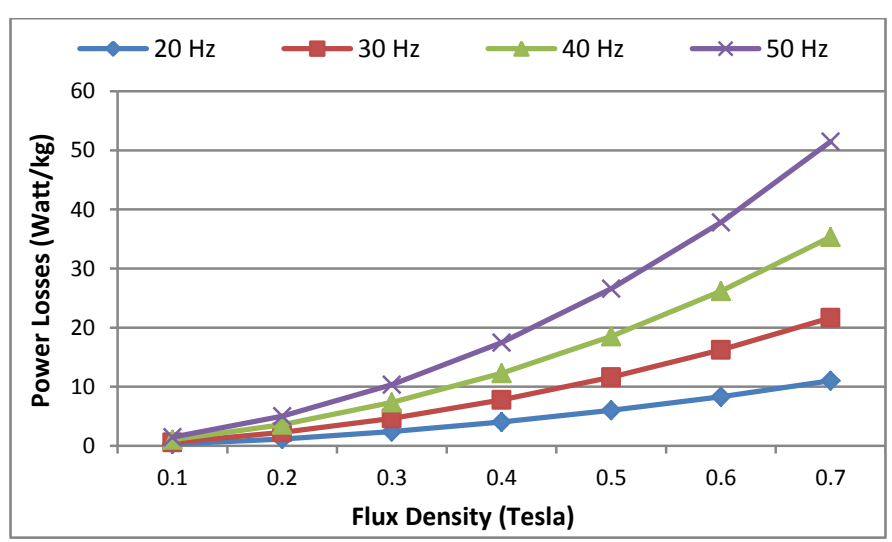

Fig. 17. Set-up 1: Ring power losses from experimental set-up

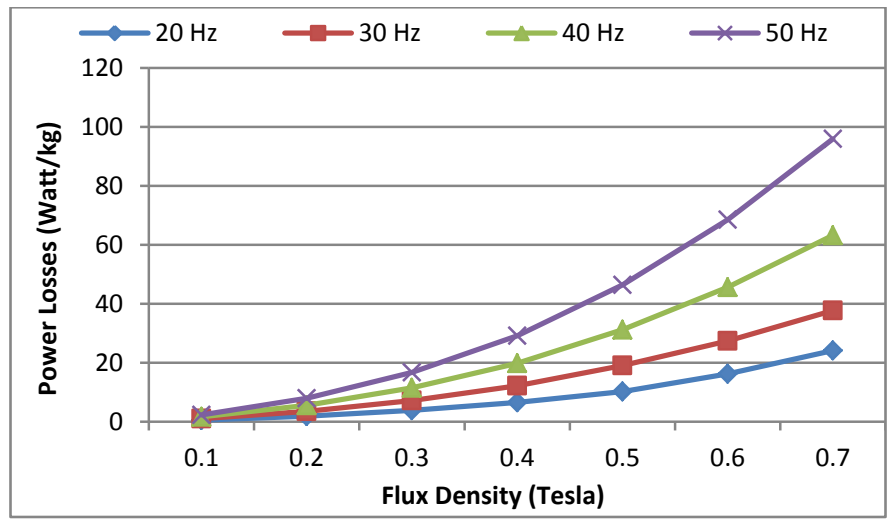

Fig. 18. Set-up 2: Ring power losses from experimental set-up

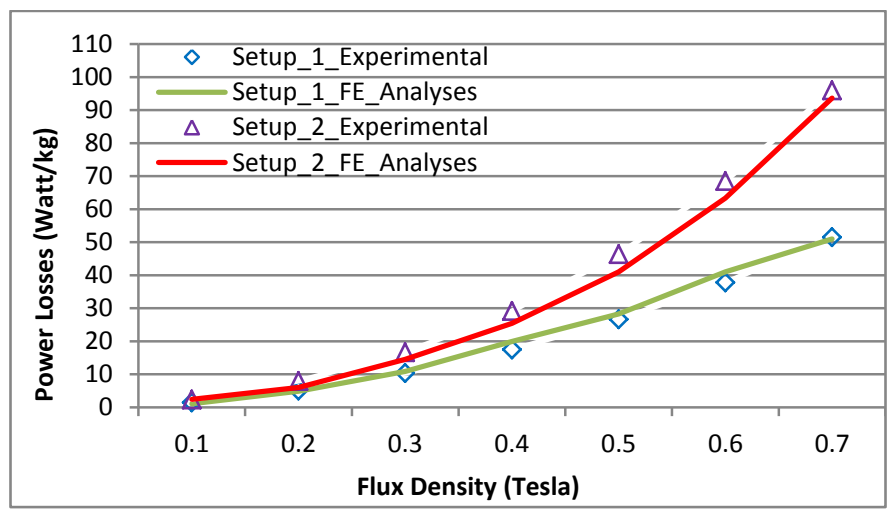

Fig. 19. FE and Experimental power losses for proposed methods at $50 \mathrm{~Hz}$ 


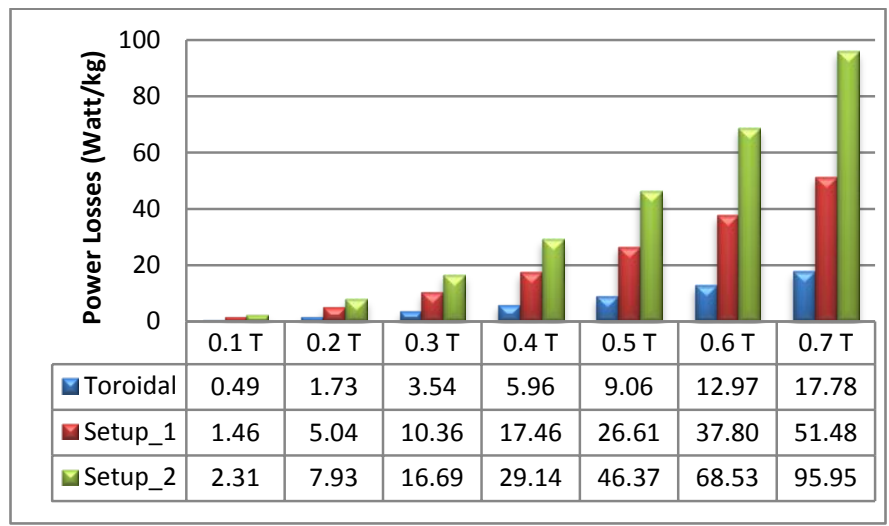

Fig. 20. Comparison of power losses for Toroidal, Set-up 1 and Set-up 2 , with different flux density at $50 \mathrm{~Hz}$.

\section{CONCLUSION}

In this paper, a new method of magnetic material characterization was proposed which provides more accurate results for losses prediction and accurate characterization of solid magnetic materials used in unconventional electrical machines.

The new method significantly improves on the conventional method by its ability to map the full losses developed from non-standard flux and eddy current behavior, which have been shown to be significantly higher than predicted by existing methods.

The implementation of this technique has also highlighted the significant under-estimation of losses that the 'classical', toroidal ring method provides with non-standard flux and eddy current distribution.

As a final remark on the proposed methods, it is clear that significant eddy current loss is identified when using Set-up 2, with a much lower loss resulting from Set-up 1. Both methods however result in significant improvements over the classical toroidal ring method.

In terms of future work, some improvements on the current set-up have been already identified. One of the first measures to be considered is the replacement of the 'low saturation', ferrite cores with higher permeability materials. This will permit testing of a wider range of magnetic materials and generating full magnetization curve.

The accurately-modelled materials are also being used for the design of a new prototype of the TLPM motor. This will be presented in future work.

\section{REFERENCES}

S. Sprague, "Lamination steels third edition A compendium of lamination steel alloys commonly used in electric motors," in The Electric Motor Education and Research Foundation, South Dartmourth, MA, USA, ed, 2007.

[2] M. Galea, G. Buticchi, L. Empringham, L. de Lillo, and C. Gerada, "Design of a High-Force-Density Tubular Motor," Ieee Transactions on Industry Applications, vol. 50, pp. 2523-2532, JulAug 2014.

[3] A. Mohammed, M. Galea, T. Cox, and C. Gerada, "Considerations for improved design and reduced manufacturing complexity of a PM, Tubular Linear Motor," in International Symposium on Linear Drives for Industry Applications, Aachen, Germany, 2015.
[4] A. J. Clerc and A. Muetze, "Measurement of Stator Core Magnetic Degradation During the Manufacturing Process," Ieee Transactions on Industry Applications, vol. 48, pp. 1344-1352, JulAug 2012.

[5] B. Standard, "Methods of measurement of the magnetic properties of magnetically soft metallic and powder materials at frequencies in the range $20 \mathrm{~Hz}$ to $200 \mathrm{kHz}$ by the use of ring specimens," in $B S$ EN 60404-6, ed: BSI, 2003.

[6] B. Measurements, "Manual for software operation of the MPG 200 D," ed: Brockhaus Measurements, 2014.

[7] D. Peckner and I. M. Bernstein, Handbook of stainless steels: McGraw-Hill New York, NY, 1977.

[8] M. Galea, C. Gerada, T. Raminosoa, and P. Wheeler, "A Thermal Improvement Technique for the Phase Windings of Electrical Machines," Ieee Transactions on Industry Applications, vol. 48, pp. 79-87, Jan-Feb 2012.

[9] N. Bianchi, S. Bolognani, D. Dalla Corte, and F. Tonel, "Tubular linear permanent magnet motors: An overall comparison," Ieee Transactions on Industry Applications, vol. 39, pp. 466-475, MarApr 2003.

[10] R. J. Tilley, Understanding solids: the science of materials: John Wiley \& Sons, 2004.

[11] W. Soong, "Bh curve and iron loss measurements for magnetic materials," Power Engineering Briefing Note Series, 2008.

[12] B. D. Cullity and C. D. Graham, Introduction to magnetic materials: John Wiley \& Sons, 2011.

[13] P. K. Lee, K. C. Kuo, C. J. Wu, Z. T. Wong, and J. Y. Yen, "Prediction of iron losses using the modified Steinmetz equation under the sinusoidal waveform," in Control Conference (ASCC), 2011 8th Asian, 2011, pp. 579-584.

[14] J. Zhong, Y. Guo, J. Zhu, H. Lu, and J. Jin, "Development of measuring techniques for rotational core losses of soft magnetic materials," Nature Sciences, vol. 2, 2007.

[15] J. F. Gieras, Permanent Magnet Motor Technolog Design and Applications, 3rd ed. U.S.A: CRC, Taylor and Francis Group, 2010.

[16] Magnetics. (2000, 01-06-2015). Ferrite Core. Material Selection Guide.

[17] J. Jamali, "End effect in linear induction and rotating electrical machines," Ieee Transactions on Energy Conversion, vol. 18, pp. 440-447, Sep 2003.

[18] J. B. Wang, W. Y. Wang, and K. Atallah, "A Linear PermanentMagnet Motor for Active Vehicle Suspension," Ieee Transactions on Vehicular Technology, vol. 60, pp. 55-63, Jan 2011.

Ahmed Mohammed received the B.Sc. degree and M.Sc. degree from the University of Technology, Baghdad, Iraq, in 1995 and 2006 respectively, both in electrical and electronic engineering. $\mathrm{He}$ is currently working toward the $\mathrm{PhD}$ degree in electrical engineering at the University of Nottingham, U.K. His research interests include high power density electrical machines design, electromagnetic actuators and material characterization of electric machines.

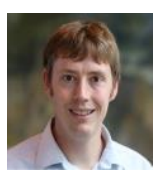

Tom Cox received his $\mathrm{PhD}$ in the development of novel linear machines from The University of Bath, UK, in 2009. He subsequently worked in industrial research and development on linear drive systems, electromagnetic actuators and electromagnetic launch systems for aircraft. In 2014 he was appointed as an Assistant Professor in electrical machines at the University of Nottingham, UK. His main research interests include the design and development of electromagnetic launch systems and actuators, integrated machine and drive systems and novel winding configurations.

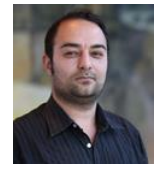

Michael Galea (M'13) received his PhD in electrical machines design from the University of Nottingham, UK, where he has also worked as a Research Fellow. He is currently a Lecturer in Electrical Machines and Drives within the PEMC research group of the University of Nottingham. $\mathrm{He}$ is the Deputy Director of the Institute for Aerospace Technology at the University of Nottingham, where he is also a Lecturer in Aerospace Systems Integration and where he manages a 
number of diverse projects related to the more / all electric aircraft and associated fields. His main research interests are design, analysis and thermal management of electrical machines and drives and the more electric aircraft.

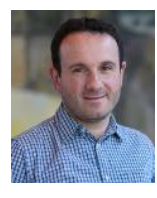

Chris Gerada (M'05) received the Ph.D. degree in numerical modelling of electrical machines from The University of Nottingham, Nottingham, U.K., in 2005. He subsequently worked as a Researcher with The University of Nottingham on high-performance electrical drives and on the design and modelling of electromagnetic actuators for aerospace applications. Since 2006, he has been the Project Manager of the GE Aviation Strategic Partnership. In 2008, he was appointed as a Lecturer in electrical machines; in 2011, as an Associate Professor; and in 2013, as a Professor at The University of Nottingham. His main research interests include the design and modelling of high-performance electric drives and machines. Prof. Gerada serves as an Associate Editor for the IEEE TRANSACTIONS ON INDUSTRY APPLICATIONS and is the Chair of the IEEE IES Electrical Machines Committee. 